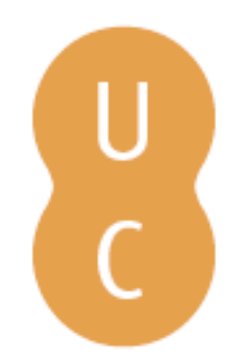

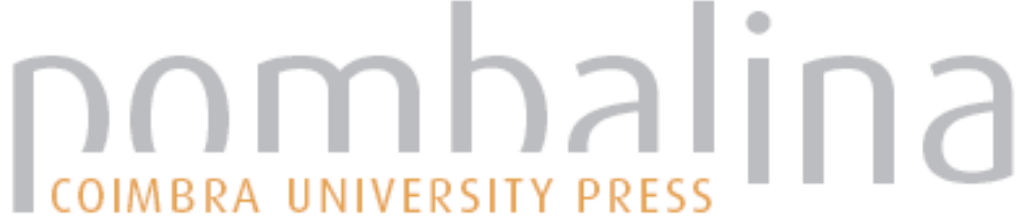

\section{Les Pheniciens entre terres et mer}

Autor(es): $\quad$ Rouillard, Pierre

Publicado por: Imprensa da Universidade de Coimbra

URL persistente:

URI:http://hdl.handle.net/10316.2/38343

DOI:

DOI:http://dx.doi.org/10.14195/978-989-26-0438-1_3

Accessed : $\quad$ 26-Apr-2023 16:17:51

A navegação consulta e descarregamento dos títulos inseridos nas Bibliotecas Digitais UC Digitalis, UC Pombalina e UC Impactum, pressupõem a aceitação plena e sem reservas dos Termos e Condições de Uso destas Bibliotecas Digitais, disponíveis em https://digitalis.uc.pt/pt-pt/termos.

Conforme exposto nos referidos Termos e Condições de Uso, o descarregamento de títulos de acesso restrito requer uma licença válida de autorização devendo o utilizador aceder ao(s) documento(s) a partir de um endereço de IP da instituição detentora da supramencionada licença.

Ao utilizador é apenas permitido o descarregamento para uso pessoal, pelo que o emprego do(s) título(s) descarregado(s) para outro fim, designadamente comercial, carece de autorização do respetivo autor ou editor da obra.

Na medida em que todas as obras da UC Digitalis se encontram protegidas pelo Código do Direito de Autor e Direitos Conexos e demais legislação aplicável, toda a cópia, parcial ou total, deste documento, nos casos em que é legalmente admitida, deverá conter ou fazer-se acompanhar por este aviso. 
Francisco de Oliveira

Pascal Thiercy

Raquel Vilaça

Coordenação

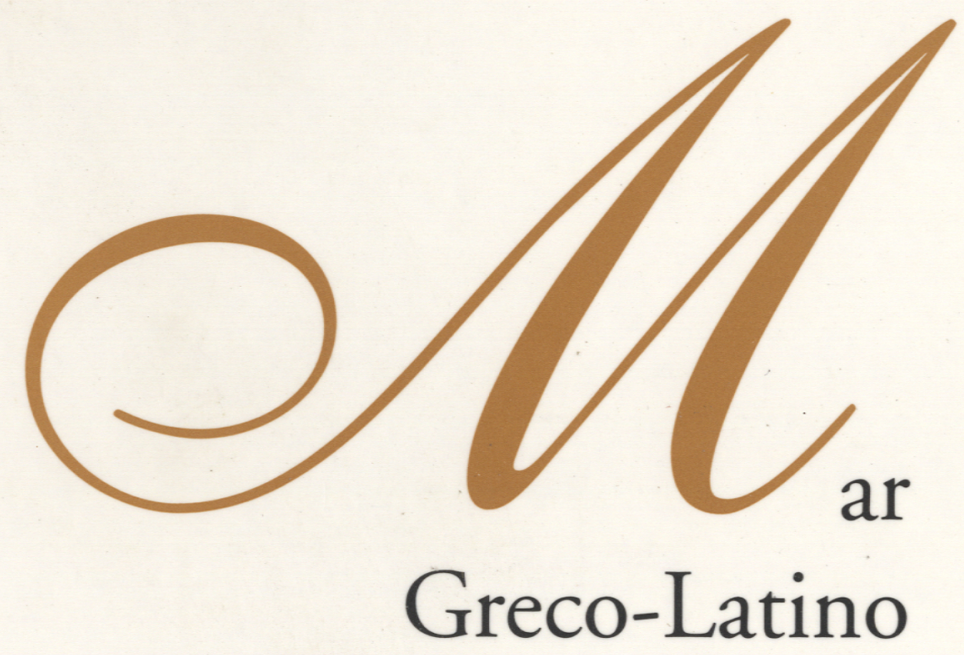

- colmbra 2006 


\section{LES PHENICIENS ENTRE TERRES ET MER}

Pierre Rouillard

CNRS - Paris

Envisager les questions des relations que les Phéniciens ont entretenu avec la terre est une des nombreuses approches possibles pour tenter de comprendre les rapports qui ont existé entre marchands méditerranéens et populations autochtones que, il y a un temps, on appelait " périphériques". Face à l'ampleur du dossier, l'esquisse de la réflexion privilégie l'époque archaïque, les $\mathrm{VIII} / \mathrm{VI}^{\mathrm{e}}$ siècles, et plutôt la Méditerranée occidentale, et ce, d'autant plus qu'une coupure fondamentale intervient au début du $\mathrm{V}^{\mathrm{e}}$ siècle avec la bataille d'Himère. Les débats sont anciens, parfois vifs, mais toujours présents comme en témoignent tant les analyses conduites dans la péninsule Ibérique que les contributions à des congrès récents, qu'il s'agisse des Congrès d'Études phéniciennes et puniques ou de la rencontre de Valence qui, en 2000, avait précisément pour thème l'agriculture dans le monde phénicien et punique, ou de celle de Mérida, en 2003, sur a El periodo orientalizante" qui, immanquablement, a touché la situation des établissements phéniciens, comme tout débat sur Tartessos envisage ces mêmes établissements.

De fait, les débats se déplacent, ou devraient se déplacer. Sans doute, s'agit-il de réfléchir sur la place que la terre (les terres quand on envisage plusieurs régions géographiques) et donc, pour une partie, l'agriculture 
occupent dans le processus d'expansion phénicienne ${ }^{(1)}$. Mais ceci conduit et conduira à envisager les relations que les Phéniciens ont entretenu avec les autochtones, en centrant nos réflexions sur les modalités de production dans un espace réputé agricole, et, immanquablement, sur les étapes préalables.

La question de la terre est presque aussi ancienne que l'étude des Phéniciens en Occident. Georges Bonsor, en effet, a intitulé en 1899 un mémoire "Les colonies agricoles pré-romaines de la vallée du Bétis "(2). Se fondant sur la découverte de tombes à incinération et à inhumation contenant bon nombre de pièces orientalisantes il en conclut que des Phéniciens étaient installés loin du rivage gaditan (à cette époque on ignorait tout des établissements côtiers de Malaga ou Grenade), dans les collines des Alcores à l'Est de Séville.

Les découvertes de Sardaigne, autour de Sulcis, de Tharros ou de Cagliari, par exemple, ont donné quelque nouveau crédit à cette thèse. Pourtant ceci ne vaut pas pour l'époque archaïque, car aucune ferme ou toute autre installation rurale n'a été reconnue et jusqu'à la fin du VI $\mathrm{VI}^{\mathrm{e}}$ siècle on ne connaît qu'une diffusion d'objets orientaux ou orientalisants dans les nuraghes ${ }^{(3)}$.

La réflexion sur le monde oriental lui même a contribué à nourrir cette hypothèse, notamment quand est souligné l'impact de la pression assyrienne qui aurait provoqué des déplacements de population phénicienne. Ce point est au cœur de l'analyse de C. R. Whittaker ${ }^{(4)}$, analyse approfondie et développée par C. G. Wagner et J. Alvar( ${ }^{(5)}$.

\footnotetext{
(1) Dossier très complet: J.-P. MOREL, Quelques remarques sur l'économie phénico-punique dans ses aspects agraires.

(2) G. BONSOR, Les colonies agricoles pré-romaines de la vallée du Bétis.

(3) M. Gras, P. Rouillard et J. Teixidor, L'univers phénicien, pp. 94-95. M. Almagro Gorbea, El indigena, pp. 249-252.

(4) C. R. WhITTAKER, Carthaginian imperialism.

(5) C. R. WAGNer, Approximación. Id. et J. Alvar, La colonización agrícola.
} 
Ces derniers ont exprimé leur conviction de l'existence d'une colonisation agraire, d'une "territorialité "(6), entendue, au moins dans un premier temps de leur travail ${ }^{(7)}$, comme un espace de domination des établissements côtiers, et pas seulement comme un " ancrage ", pour reprendre la formule " moyenne " de Jean-Paul Morel ${ }^{(8)}$, dans la terre, la production et la commercialisation des produits de la terre.

Il convient de reprendre les principaux points de débats qui tournent souvent autour du vocabulaire même, des ressorts des navigations phéniciennes, des grandes phases et de la géographie de l'expansion phénicienne.

Dans un milieu académique marqué par les études classiques, il est courant d'avoir recours à un vocabulaire et à des concepts forgés pour le monde gréco-romain, qui, eux-mêmes, ont connu des vicissitudes au cours de notre histoire politique. Tel est le cas du mot "coloniser" qui lui même ne s'est imposé qu'aux $\mathrm{XVII}^{\mathrm{e}}$ et $\mathrm{XVIII}^{\mathrm{e}}$ siècles ${ }^{(9)}$, conçu par des latinistes, pour le monde romain, et repris pour le monde grec. Il est lié au verbe colere qui signifie cultiver et le mot "colonie" indique un type d'installation romaine, avec - à l'époque républicaine - des aspects militaires; ensuite - sous l'Empire - le statut de colonie est le statut le plus haut auquel pouvait aspirer une cité, quelles qu'aient été son origine et son histoire précédente. Le mot des Grecs, apoikia ("le fait d'habiter à distance"(10)) n'a pas été utilisé dans notre parler courant qui privilégie les mots de la famille du mot "colonie". Ainsi l'usage du mot "colonisation" s'est-il répandu, avec la connotation que

\footnotetext{
(6) Citons la phrase de C. G. WAGner et J. Alvar : a La territorialidad se convierte en un elemento básico de las colonias fenicias junto con el aprovechamiento de los recursoss agrícolas locales , dans Fenicios en Occidente, p. 101.

(7) Position plus prudente, sans faire, au moins dans le titre de l'article, l'économie du mot a colonie , dans C. G. WaGner et J. Alvar, La colonización agricola.

(8) J. P. MOREL, Quelques remarques sur l'économie phénico-punique, p. 418.

(9) E. LEPORE, La Grande Grèce, aspects et problèmes d'une “colonisation" ancienne, pp. 17-36 (notamment pp. 17-18); M. CASEVITZ, Le vocabulaire de la colonisation en grec ancien, notamment pp. 9-11 ; M. Gras, La Méditerranée archaïque, pp. 122-123.

(10) M. CASEvitz, Le vocabulaire de la colonisation en grec ancien, pp. 114-135.
} 
lui ont donnée les expériences coloniales des époques moderne et contemporaine, qui, rappelons-le, mobilisent commerçants, agriculteurs et militaires dans le but d'occuper un territoire ${ }^{(11)}$. La notion de dépendance est alors toujours présente, alors que ce n'était pas le cas des "colonies" grecques (en dehors des archai). Puis "colonisation" a suscité "précolonisation", terme d'abord utilisé pour le monde grec et que l'on retrouve souvent repris pour le monde phénicien.

Doit-on en conclure que les Phéniciens ont fondé des "colonies" comparables à celles des Romains ou à celles des temps modernes? Un non vigoureux ne sera pas suffisant pour corriger quelques abus de langage.

Dans la littérature scientifique relative aux Phéniciens d'Occident, les mots "colonie" et "colonisation" sont encore, malheureusement, l'objet d'un usage généralisé et ambigu. Mais un courant critique se dessine aujourd'hui à l'encontre du mot "précolonisation". Certes la référence à Moscati semble oubliée $^{(12)}$, mais l'analyse des données met à leur juste place ces "odyssées", ces contacts, brefs, de quelques décennies, caractérisés par des échanges (souvent sous forme de troc, de don, de cadeau) sans occupation de territoire, qui peuvent exister mais ne sont pas des préalables nécessaires à une installation $^{(13)}$.

Les ressorts des mouvements phéniciens, sont, d'abord le commerce; la perception du phénomène de l'expansion phénicienne a été, et est souvent

\footnotetext{
(11) Le mot "colonialisme" est - malheureusement - trop souvent utilisé lui aussi par les archéologues, sans que sa définition actuelle (contrôle stratégique et financier des points névralgiques d'un territoire, nécessitant peu de monde) soit nuancée, adaptée voire corrigée.

(12) S. MOSCATI, Precolonizzazione greca e precolonizzazione fenicia; M. GRAS, P. ROUILLARD et J. TEIXIDOR, L'univers phénicien, pp. 110-111.

${ }^{(13)}$ Pour le monde grec, voir E. LEPORE, La Grande Grèce, aspects et problèmes d'une "colonisation" ancienne, notamment p. 27. Sur le monde phénicien d'Occident, voir les positions nuancées suivantes : $\mathrm{M}^{\mathrm{a}} \mathrm{E}$. AuBET, Tiro y las colonias fenicias de Occidente, pp. 185187 ; D. Ruiz Mata, dans M. Almagro Gorbea et alii, Protobistoria de la Peninsula Ibérica, pp. 67-69 ; J. AlvAR, Comercio e intercambio en el contexto precolonial ; C. G. WAGNER, Comercio lejano, colonización e intercambio desigual en la expansión fenicia arcaica por el mediterraneo. Les éléments "précoloniaux " sont valorisés, notamment et récemment, par M. ALMAGRO GORBEA, La "précolonización fenicia "en la Península Ibérica.
} 
encore, liée au seul commerce et le " tout commercial " a été l'explication de l'expansion phénicienne. La nécessité de ressources agricoles a été ensuite l'explication privilégiée. Aujourd'hui, la pêche, notamment celle du thon, est présentée comme un des ressorts de l'installation comme le suggère par exemple une récente fouille à Cadix ${ }^{(14)}$, et ce dès la seconde moitié du VIII ; la même analyse est faite aussi pour Nora ${ }^{(15)}$.

L'excellence de l'agriculture punique, celle que Magon symbolise, pourrait servir aussi de fondement à une explication fondée sur l'exploitation des terres. On relèvera que cet argument, celui de l'écho de Magon, ne ressort pas dans la démarche de ceux qui ont poussé à l'extrême cette explication qui a connu les étapes évoquées déjà, avant de susciter des interrogations même au sein de ceux qui ont porté "l'hypothèse agricole "(16) ou de connaitre un changement de registre qui privilégie l'étude des modalités de rencontre entre les Phéniciens et les autres.

De fait, tout n'est pas simple quand on prend en compte quelques textes et realia. Ainsi, parle-t'on de pénurie de produits alimentaires en Orient ; mais Hiram reçoit du blé et de l'huile de Salomon (I Rois, V, 11). Le De Mirabilis Auscultationibus nous rapporte ( $\$ 135$ ) que de l'huile phénicienne était transportée vers Tartessos pour être échangée avec des métaux. Le Pseudo-Skylax (\$112) rapporte que les « Ethiopiens " (de Lixus) font beaucoup de vin de leurs vignes que les Phéniciens exportent.

Mais, à partir de cette observation tout se bouscule et les interrogations s'enchaînent. On compte peu d'amphores orientales en Occident ; et là, les enquêtes de Joan Ramon sont d'un apport essentiel ${ }^{(17)}$. Le texte du Pseudo-Aristote vaut-il pour l'époque archaïque ? Le vin, produit autour de Lixus, estil le résultat d'un apprentissage par les Phéniciens?

\footnotetext{
(14) I. CORdoba Alonso et D. Ruiz MATA, El asentamiento fenicio arcaico de la calle Cánovas del Castillo (Cádiz).

(15) M. BOTTO et alii, Nora, p. 182.

(16) C. G. WaGner et J. Alvar, La colonización agricola, p. 201.

(17) J. RAMON, Las ánforas fenicio-púnicas.
} 
Le danger de l'histoire régressive est bien là. Les textes sur l'agriculture à Carthage sont des $\mathrm{IV}^{\mathrm{e}} / \mathrm{II}^{\mathrm{e}}$ siècles avant J.- C. et valent pour la période qui suit la bataille d'Himère ; par exemple quand les soldats d'Agathocle admirent les jardins du cap Bon, soit vers 310 av. J.-C. (Diodore, XX, 8, 35). Un autre témoignage des capacités que recelait Carthage nous est fourni quand cette cité, à la fin du VI ${ }^{\mathrm{e}}$ siècle, fait couper les plantes fruitières en Sardaigne ; mais - de manière complémentaire - le texte du Pseudo-Aristote (§ 100) qui relate cet événement suggère bien sûr l'existence en Sardaigne de cultures arboricoles, celles probables de l'olivier et de la vigne, dont l'ampleur a pu devoir beaucoup aux Phéniciens.

Avant le $V^{e}$ siècle, avant la conquête d'un vaste espace, qu'en est-il à Carthage même? On sait seulement que les Carthaginois payaient un loyer aux « Lybiens ». Et aucune ferme n'est connue pour l'époque archaïque.

Déplacer notre regard semble nécessaire, une fois rappelées quelques données relatives à l'exploitation de la terre. L'observation des pratiques agricoles ne montre pas, au moins dans la péninsule Ibérique, de grandes différences entre celles que l'on attribue aux Phéniciens et celles attribuées aux indigènes. Les possibilités de mise en culture sont davantage mises à profit à l'époque archaïque, mais, ceci vaut dès le Bronze Final, ce qui, très probablement, a attiré les Phéniciens ${ }^{(18)}$. L'existence de choix propres aux Phéniciens est admise, mais ils sont connus pour avoir été mis en œuvre par les Puniques, à partir du $\mathrm{V}^{\mathrm{e}}$ siècle, notamment l'arboriculture, la vigne et l'olivier ${ }^{(19)}$.

Pour passer de l'étude de la "territorialité " aux modes de rencontre, le point de départ de mon analyse réside dans le constat que les espaces de production fonctionnent seulement quand les Phéniciens s'établissent. Ainsi, avant de regarder les terres de l'arrière pays, regardons de manière prioritaire

\footnotetext{
${ }^{(18)}$ P. Iborra, E. Grau et G. Perez Jorda, Recursos agrícolas, p. 49. F. Lopez Pardo et J. Suarez PADILla, Aproximación al conocimiento del paleoambiente, p. 78.

(19) J.-P. MOREL, Quelques remarques sur l'économie phénico-punique dans ses aspects agraires.
} 
la situation sur le littoral, au travers de quatre exemples, situés aux embouchures de trois rivières, le Guadalhorce, le Guadalmedina, l'Almanzora et le Segura.

À l'embouchure du Río Guadlhorce ${ }^{(20)}$, les terres sont libres au Bronze Final et les habitats indigènes sont dans les collines qui entourent les vallées secondaires des affluents du Guadalhorce. Si les Phéniciens qui s'installent à la fin du VIII ${ }^{\mathrm{e}}$ siècle ne cultivent pas au départ cela ne tient pas à une pression indigène gênante; en s'installant sur le Cerro del Villar, ils ne viennent pas non plus d'abord pour cultiver. Le grand changement intervient courant $\mathrm{VII}^{\mathrm{e}}$ siècle, avec, d'une part, un phénomène d'érosion des sols suite à une surexploitation des terres - sous contrôle indigène- qui entourent la dépression du Guadalhorce, et d'autre part, plutôt au VI ${ }^{\mathrm{e}}$ siècle, avec l'apparition de communautés rurales comme sur la Loma del Aeropuerto.

Ceci vaut aussi pour Malaga : les indigènes s'établissent à San Pablo sur les bords de l'embouchure du Guadalmedina dans le même temps que s'établissaient les Phéniciens à Malaka ${ }^{(21)}$.

Dans la région de l'embouchure de l'Almanzora, les études paléobotaniques ont montré une continuité des espèces cultivées et de leur proportion entre elles, dans la dépression de Vera et dans l'environnement immédiat des établissements phéniciens. Ici c'est, comme d'ailleurs au Cerro del Villar, l'orge qui domine. Ceci témoigne de l'association, de l'intégration d'autochtones aux étrangers établis sur le littoral : il y a, selon José Luis López Castro, " adaptation " des " colons " phéniciens à la situation locale, un fait que nous considérons essentiel. Toutefois notre collègue soutient qu'ont existé (alors, en plus de la mise culture par les indigènes ?) des " asentamientos rurales " phéniciens, pour lesquels manquent - pourtant - des précisions chronologiques ${ }^{(22)}$.

\footnotetext{
${ }^{(20)}$ M. E. Aubet et alii, Cerro del Villar-1. M. E. AubeT et A. Delgado, La colonia fenicia del Cerro del Villar.

${ }^{(21)}$ F. LÓPEZ PARdo et J. Suarez Padilla, Aproximación al conocimiento del paleoambiente, p. 81.

(22) J. L. LÓPEZ CASTRO, Baria, pp. 102-103.
} 
Le cas de Guardamar del Segura ${ }^{(23)}$ nous a servi de point de départ pour une nouvelle perception de la relation entre marchands étrangers et autochtones. À l'embouchure du Segura, au sud de la ville d'Alicante, La Rabita/Fonteta, dans la commune de Guardamar del Segura, se trouve en aval de sites nés à l'âge du Bronze, dans le Sierra de Crevillente comme Peña Negra qui a livré un assez abondant matériel orientalisant, et de sites qui ont livré, dès la fin de l'Ibérique ancien, des sculptures de claire filiation orientale comme Agost, Redovan, Elche, et même, plus loin vers le Nord, Pozo Moro. La basse vallée du Segura ne saurait être lue indépendamment de ce grand centre de peuplement de la Sierra de Crevillente au nord de l'embouchure du Segura.

Ce site peut être qualifié de "portuaire ", car il possède à tout le moins un débarcadère, à l'arrière d'une barre; un débarcadère comblé au VI ${ }^{\mathrm{e}}$ siècle. L'originalité de cet habitat tient d'abord à sa situation côtière, sur une plage. Dans la première moitié du dernier millénaire ceci est exceptionnel sur ce littoral oriental de la Méditerranée, à l'exclusion des installations " étrangères ". Or là, dans le dernier quart du VIII ${ }^{\mathrm{e}}$ siècle, sur l'ensemble du matériel, $70 \%$ est indigène, façonné à la main, 20 \% est constitué d'amphores et le reste de vaisselle fine, pour partie phénicienne. Ensuite, aux $\mathrm{VII}^{\mathrm{e}} / \mathrm{VI}^{\mathrm{e}}$ siècles le pourcentage de vaisselle faite à la main restera stable aux alentours de $35 \%$ et la céramique à vernis rouge aux alentours de $20 \%$. Ainsi, sommes nous en présence d'un habitat où se confrontent l'importance de la présence indigène et les options des navigateurs phéniciens qui se retrouvent associés en un même établissement.

Il est, dès lors, loisible d'analyser innovations et ruptures, liées à cette confrontation ou cette cohabitation. Innovation avec la première attestation dès le début du VII ${ }^{\mathrm{e}}$ siècle - des maisons complexes à pièces rectangulaires, de claire filiation orientale ${ }^{(24)}$, bâties en pisé avec ou sans solin maçonné et

\footnotetext{
(23) P. ROUILLARD et alii, Fouilles de La Rabita de Guardamar del Segura (Alicante), II.

(24) F. BRAEMER, L'architecture domestique du Levant à l'Âge du fer, pp. 66, 68, 104.
} 
avec des sols chaulés et peints. Ces innovations font de Rabita/Fonteta, au $\mathrm{VII}^{\mathrm{e}}$ siècle - un habitat phénicien. Ruptures avec le regain, fin $\mathrm{VII}^{\mathrm{e}}$ siècle, des constructions sur poteaux porteurs, comme à la fin du VIII ${ }^{\mathrm{e}}$ siècle. Rupture, vers 600 , avec une fortification puissante, aux caractères simples, à l'appareil peu soigné ; mais ne connaissant pas de porte ou de passage, seul lieu d'une possible mise en œuvre de techniques élaborées, nous ne pouvons pas proposer une filiation. A partir de cette époque, au $\mathrm{VI}^{\mathrm{e}}$ siècle, les maisons ont une ou deux pièces, et sont alignées le long du mur : ceci sera la norme des habitats ibériques anciens. Ici, nous sommes en présence de ce qui peut être lu comme un régression, ou une expérimentation. Et, à cette même époque, un ou des monuments (funéraires, cultuels ?), connus seulement par des blocs épars issus de ce ou ces monuments et utilisés en remploi, comme éléments de construction de la Rabita califale, qui sont probablement le témoignage le plus ancien d'architecture orientalisante de la Péninsule.

Rabita/Fonteta est plus un site d'échange que de production, car nous disposons de peu d'indices permettant de penser que cette communauté ait dépassé le stade de l'autoconsommation. Les traits de l'agriculture sont apparemment conformes aux pratiques de la région. Pour les bovins, on relèvera le faible pourcentage d'animaux de traction pour l'exploitation d'un terroir, qui - il est vrai- est pour une bonne part lagunaire. Mais, relevons aussi l'abondance des amphores locales, surtout à partir de la seconde moitié du VII ${ }^{\mathrm{e}}$ siècle: il y a donc bien des productions vivrières d'un immédiat proche. Les témoignages d'une petite métallurgie, du bronze et du fer, ne manquent pas, mais, dans l'espace que nous avons étudié, ils sont modestes, à l'image des ressources naturelles de la région. Il est dès lors difficile de conduire une réflexion sur d'éventuels surplus.

Ainsi sommes-nous en en présence d'un site choisi pour sa situation, au débouché d'un axe naturel d'échange et pour la variété des ressources agricoles et halieutiques. La communauté de Rabita/Fonteta met à profit de 
manière " opportuniste "les potentialités, géographiques et humaines, de cette région du bas Segura.

Il convient de souligner les changements observés dans les choix de conditions de vie et les comportements, autour de 700, autour de 600. Que ce soit dans l'architecture ou dans la céramique, on saisit la preuve de la réunion de deux communautés. L'évolution des pratiques alimentaires est aussi riche d'enseignements; des tendances nous paraissent sensibles, quand, dans les premières décennies du site, à la fin du VIII $^{\mathrm{e}}$ siècle, on consommait des espèces d'escargots d'origine terrestre et des poissons de rivières ; après 700, l'alimentation change, avec cette fois une préférence très marquée pour les espèces marines.

Les Phéniciens n'interviennent pas dans une région vide. Mais distinguons région et site immédiat. Le binôme, " pouvoir " de l'intérieur et lieu spécialisé sur la côte est courant dans la Méditerranée; citons seulement les cas exemplaires de Gravisca ou Pyrgi. Ici, dans cette région de la basse vallée du Segura, nous voyons une complémentarité des secteurs côtiers et montagneux: le site abrité de Rabita/Fonteta a su attirer le marchand phénicien et devenir ainsi le point de rencontre de Phéniciens et de populations de l'intérieur déjà initiées - depuis le Bronze atlantique- aux échanges. Un double mouvement s'établit: arrivée des Phéniciens et établissement de familles, venant de l'arc montagneux voisin, les deux communautés "coagulant". Ensuite se produit un phénomène de " créolisation " ${ }^{(25)}$, quand, au $\mathrm{VI}^{\mathrm{e}}$ siècle, les Phéniciens se retrouvent absorbés. C'est à ce moment que les populations locales affirment leur culture, ibérique, et, pour notre propos, font le choix d'une installation littorale. De la terre vers la mer, naissent ensuite des habitats comme Campello ou Tossal de Manises, pour ne prendre d'exemple que dans la région alicantine.

\footnotetext{
(25) Le même mot se retrouve chez M. Almagro Gorbea, El indigena, p. 244.
} 
Deux données sont à prendre en considération ensemble : les indigènes sont ceux qui, dans les sites littoraux, les établissements phéniciens, exploitent la terre ${ }^{(26)}$, et cette exploitation des terres littorales ne précède pas l'installation des Phéniciens.

Le terme de "coagulation" semble rendre compte de ce processus de rencontre de groupes sociaux, et nous sommes, dès lors, loin du système grec avec chora immédiate.

Identifier une culture, face à la question envisagée, est un impératif. La réponse, même dans le cas des établissements où une communauté phénicienne est attestée, reste toujours un enjeu discuté. Le cas de Lixus, en cours d'étude, est exemplaire, car il nous fournit à la fois des informations et des questionnements qu'il convient d'avoir en permanence à l'esprit. Si l'on prend en compte le critère le plus simple, sans doute aussi le plus explicite, celui du pourcentage de chacune des catégories de céramiques, comme nous l'avons fait par exemple pour Guardamar del Segura, les informations fournies par les fouilleurs de Lixus suscitent le débat le plus nourri possible. A Lixus : le pourcentage de la vaisselle faite à la main dans les niveaux les plus anciens, dans les remblais préalables aux premières constructions phéniciennes, oscille entre $36 \%{ }^{(27)}$ et $43 \%{ }^{(28)}$ Pourtant l'antériorité de la présence indigène n'est pas assurée, car les collègues qui analysent ces céramiques façonnées à la main formulent plusieurs hypothèses, toutes méritant d'être prises en considération. Au delà d'un premier débat, céramique façonnée à la main des indigènes du lieu/ou faite sur place par les Phéniciens ${ }^{(29)}$, est formulée l'hypothèse d'une série céramique portée par les

\footnotetext{
(26) F. LÓPEz PARDo et J. SuAREz PADIlla, Aproximación al conocimiento del paleoambiente, pp. $78-81$.

${ }^{(27)}$ M. Habibi et alii, dans C. Aranegui (dir.), Lixus-2, p. 176.

${ }^{(28)}$ N. Alvarez et alii, dans C. Aranegui (dir.), Lixus-1, p. 74.

(29) M. BElen et alii, dans C. Aranegui (dir.), Lixus-1, p. 83-89.
} 
Tartessiens, les populations du nord du Détroit ${ }^{(30)}$, qui auraient accompagné les Phéniciens au moment de leur installation à l'embouchure du Loukos. Le cas de Lixus est exemplaire de l'éventail des différentes situations possibles, étant entendues, d'une part, la grande parenté des céramiques façonnées à la main du Bronze final ou de l'époque orientalisante du sud de la Péninsule et celles mise au jour à Lixus et, d'autre part, la médiocre connaissance que nous avons des artisanats céramiques de la partie septentrionale de l'actuel Maroc ; mais une donnée semble à peu près acquise, celle d'une contemporanéité de l'installation en un même lieu de Phéniciens et de populations que - faute de précisions- nous pouvons qualifier de " régionales".

La question de la présence de communautés phéniciennes dans les profondeurs des terres andalouses peut être abordée par une - simplecartographie des sites ayant reçu de telles communautés. La géographie de ce que l'on peut résumer sous le vocable de "signes phéniciens ", fournit un dessin que l'on peut superposer au contour de ce que l'on appelle le golfe "tartessique ". On ne saurait expliquer la présence de témoignages orientaux par la présence de groupes de Phéniciens installés dans les terres indigènes, car la carte montre clairement que les sites en question ont été - à l'époque archaïque - des habitats côtiers, sur les marges du golfe "tartessique "(31).

Les modes de relations sociales ${ }^{(32)}$, les rapports sociaux ou les types de sujétion nous sont pour l'essentiel inconnus à La Rábita/Fonteta, comme ailleurs, mais on voit mal comment on peut sortir d'une relation établie en termes d'“ amitié” ou d'“ hospitalité", même si l'usage de ces mots n'est assorti d'aucune précision quand à la forme de l'entente. Ces traits sont partie prenante d'un emporion, dont les premiers jalons ont pu être constitués

\footnotetext{
(30) N. Alvarez et alii, dans C. Aranegui (dir.), Lixus-1, p. 73-82. Ce dossier est repris, sans dépasser le débat ouvert, par M. HABiBI et alii, dans C. Aranegui (dir.), Lixus-2, p. 176-178 et M. Almagro, El indígena, p. 243.

${ }^{(31)} \mathrm{J}$. L. ESCACENA et R. IZQuiERdo, Oriente en Occidente, p. 124. R. IzQuiERdo de MONTES et G. FERNÁNDEZ TRONCOSO, Del poblamiento de época orientalizante, p. 715.

${ }^{\left({ }^{32}\right)}$ La lecture de J. Alvar, Comercio e intercambio en el contexto precolonial, est essentielle.
} 
d'activités saisonnières. Après avoir jugé inadéquat le terme de "colonie" il convient alors de proposer une définition pour la communauté de La Rábita/Fonteta : "un emporion entre Ibères et Phéniciens", même si le mot est grec et même si pour un Grec la présence d'un partenaire grec y est nécessaire ; si nous l'utilisons ici, c'est parce que ce concept est celui qui rend le mieux compte par ses mécanismes de ce que nous avons observé sur le terrain. Cette formulation est d'ailleurs plus adéquate pour le VII siècle que pour le $\mathrm{VI}^{\mathrm{e}}$ siècle qui voit les Phéniciens progressivement absorbés par le fonds de population indigene ${ }^{(33)}$. Le poids du milieu indigène, immédiatement présent et assumant le pouvoir localement, est exprimé dans ces formules et il ne paraît pas aventureux d'envisager d'étendre à bien des établissements cette analyse et, en se risquant à une histoire régressive, de proposer une symétrie entre le binôme Alcudia de Elche - La Picola de Santa Pola qui fonctionna un siècle environ entre le milieu du $\mathrm{V}^{\mathrm{e}} \mathrm{s}$. et le milieu du $\mathrm{IV}^{\mathrm{e}} \mathrm{s}$. et celui formé par Peña Negra II et La Rábita pour le VII et le début du VI ${ }^{\mathrm{e}}$ s. av. J.- $\mathrm{C}^{(34)}$. Entre la mer et les terres, les relations sont complexes et ne nous apparaissent en aucun cas comme unilatérales.

\footnotetext{
${ }^{(33)}$ La région de la basse vallée du Segura en offre un bel exemple, quand à El Molar, Feliciana Sala, a identifié, parmi des tombes ibériques à incinération, une tombe (peut-être deux) à inhumation, de claire filiation punique : voir F. SALA, Algunas reflexiones sobre la fase antigua de la Contrestania, pp. 19-20.

(34) P. ROUIllard, Emporion et emporia.
} 


\section{BIBLIOGRAPHIE}

\section{Abréviations :}

CIEFP IV = Actas del IV Congreso International de Estudios Fenicios y Púnicos (Cádiz, 1995), Cadix, 2000.

El periodo orientalizante $=$ Sebastián CELESTINO PÉREz et Javier JimÉnEz Ávila (éds.), El periodo orientalizante, Actas del III Simposio Internacional de Arqueología de Mérida : Protobistoria del Mediterraneo Occidental (Mérida, 2003), Anejos de AEspA, XXXV, Mérida, 2005.

GÓmez Bellard (éd.), Ecohistoria = Carlos GÓmEz Bellard (éd.), Ecohistoria del paisaje agrario, La agricultura fenicio-púnica en el Mediterráneo (Valencia, 2000), Valence, 2003.

\section{Monographies et articles :}

Almagro-Gorbea, Martín, a La a precolonización fenicia s en la Península Ibérica *, dans IV CIEPP, Vol. II, 2000, pp. 711-721.

Almagro-Gorbea, Martín et alii, Protohistoria de la Península Ibérica, Madrid, 2001.

Almagro-Gorbea, Martín, Mariano TORRes et Alfredo Mederos, a El indígena ", dans José A. ZAMORA (éd.), El bombre fenicio, Estudios y materiales, Rome, 2003, pp. 241-255.

Alvar, Jaime, Comercio e intercambio en el contexto precolonial ", dans Intercambio y Comercio Preclásico en el Mediterraneo, I Coloquio del CEFYD (Madrid, 1998), Madrid, 2000, pp. 27-34.

ARANEgui, Carmen (dir.), Lixus-1, Colonia fenicia y ciudad púnico-mauritana, Anotaciones sobre su ocupación medieval, Saguntum, Extra-4, Valence, 2001.

ARANEGUI, Carmen (dir.), Lixus-2, Ladera Sur, Excavaciones arqueológicas marroco-españolas en la colonia fenicia, Campañas 2000-2003, Saguntum, Extra-6, Valence, 2005.

AuBET, M $M^{a}$ Eugenia, Tiro y las colonias fenicias de Occidente (2 è éd.), Barcelone, 1994.

Aubet, Ma Eugenia, Pilar Carmona, Elisenda Curià, Ana Delgado, Antonio Fernández Cantos et Mercedes PÁRraga, Cerro del Villar - I, El asentamiento fenicio en la desembocadura del río Guadalhorce y su interacción con el binterland, Séville, 1999.

Aubet, Ma Eugenia et Ana Delgado, a La colonia fenicia del Cerro del Villar y su territorio , dans Gómez Bellard (éd.), Ecohistoria, pp. 57-74.

Azuar, Rafael, Pierre Rouillard, Éric Gailledrat, Pierre Moret, Feliciana Sala Sellés et Alain Badie, "El asentamiento Orientalizante e Ibérico Antiguo de "La Rábita", Guardamar del Segura (Alicante). Avance de las excavaciones 1996-1998", Trabajos de Prehistoria, 55, 2, 1998, pp. 111126.

BonsOR, Georges, "Les colonies agricoles pré-romaines de la vallée du Bétis *, RA, XXXV, 1899, pp. $126-159$ et $232-391$.

BotTo, Massimo, « Rapporti fra Fenici e indigeni nella Penisola iberica (VIII-VI sec. a.C.) *, dans G. Unso, Hispania Terris Omnibus Felicior, Premesse ed esiti di un processo di intregrazione, Rome, 2002, pp. 9-62.

Botro, Massimo, Stefano FINOCCHI, Susanna Melis et Marco Rendeli, « Nora : Sfruttamiento del territorio e organizzazione del paesagio in età fenicia e punica , dans Gomez BELlaRD (éd.), Ecobistoria, pp. 151-186. 
BraEmer, Frank, L'architecture domestique du Levant à l'Âge du Fer, ADPF, Paris, 1982.

CASEVITZ, Michel, Le vocabulaire de la colonisation en grec ancien, Paris, 1985.

CóRdoba Alonso Ignacio et Diego Ruiz MATA, "El asentamiento fenicio arcaico de la calle Cánovas del Castillo (Cádiz). Un análisis preliminar", dans El periodo orientalizante, pp. 1269-1322.

DRIDI, Hédi, Carthage et le monde punique, Paris, 2006.

Escacena CARrasco, José Luis et Rocio IZQuierdo de MONTES, "Oriente en Occidente : arquitectura civil y religiosa en un "barrio fenicio " de la Caura Tartessica ", dans Diego RuIz MATA et Sebastián CELESTINO PÉREz (éds.), Arquitectura Oriental y Orientalizante en la Península Ibérica, Madrid, 2001, pp. 123-157.

Gómez Bellard, Carlos, "Agricultura fenicio-púnica : algunos problemas y un caso de estudio ", Complutum Extra, 6 (I), 1996, pp. 389-400.

Gonzalez Prats, Alfredo, Nueva luz sobre la protobistoria del Sudeste, Universidad de Alicante, Alicante, 1990.

GonZÁlez Prats, Alfredo, "Nuevos datos sobre urbanística y cultura material en el Hierro Antiguo del Sudeste (Peña Negra, 1986)", Lucentum, IX-X, 1990-91, pp. 51-75.

Gonzalez Prats, Alfredo, "La presencia fenicia en el Levante peninsular y su influencia en las comunidades indígenas ", dans I-IV Jornadas de Arqueología Fenicia-Púnica (Ibiza, 1986-1989), Ibiza, coll. “ Trabajos del Museo Arqueológico de Ibiza ” (24), 1991, pp. 109-118.

GonzÁlez Prats, Alfredo, "La Fonteta. El asentamiento fenicio de la desembocadura del río Segura (Guardamar, Alicante, España). Resultados de las excavaciones de 1996-97 ", Rivista di Studi Fenici, 26, 2, 1998, pp. 191-228.

Gonzalez Prats, Alfredo, "Los Fenicios en la fachada oriental hispana ", dans XVI Jornadas de Arquología Fenicio-Púnica (Eivissa, 2001), Ibiza, coll. "Treballs del Museu Arqueològic d'Eivissa i Formentera "(50), 2002, pp. 127-143.

Gras, Michel, La Méditerrranée archaïque, Paris, 1995.

Gras, Michel, Pierre Roulllard et Javier TeIXIDOR, L'univers phénicien, 2éd, Paris, 1995.

IBORRA, Pilar, GraU, Elena et PÉREz JORDA, Guillem, " Recursos agrícolas y ganaderos en el ámbito fenicio occidental : estado de la cuestión », dans GÓmez BELlard (éd.), Ecohistoria, pp. 33-55.

IzQuierdo de Montes, Rocio et Guiomar Fernández Troncoso, "Del poblamient de época orientalizante en Andalucía y de sus problemas ", dans El periodo orientalizante, pp. 709-730.

LEPORE, Ettore, La Grande Grèce, aspects et problèmes d'une "colonisation" ancienne, Naples, Centre Jean Bérard, coll. "Etudes "(V), 2000.

LÓPEz CASTRO, José Luis, "Baria y la agricultura fenicia en el extremo Occidente ", dans Gómez BELlard (éd.), Ecohistoria, pp. 93-110.

LÓPEZ PARdo, Fernándo et José SuÁrez PAdIlla, "Aproximación al conocimiento del paleoambiente, poblamiento y aprovechamiento de los recursos durante el primer milenio A.C. en el littoral occidental de Málaga », dans GÓmez Bellard (éd.), Ecohistoria, pp. 75-91.

MOREL, Jean-Paul, "Quelques remarques sur l'économie phénico-punique dans ses aspects agraires ", dans CIEFP IV, pp. 411-423.

MosCati, Sabatino, “ Precolonizzazione greca e precolonizzazione fenicia ", Rivista di Studi Fenici, 11, 1, 1983, pp. 1-7. 
RAMÓN, Joan, Las ánforas fenicio-púnicas del Mediterráneo central y occidental, Barcelone, 1995.

ROUILLARD, Pierre, "Emporion et Emporia : quelques observations sur linitiative et la tutelle, dans ", dans Intercambio y Comercio Preclásico en el Mediterráneo, I Coloquio del CEFYD (Madrid, 1998), Madrid, 2000, pp. 259-265.

Roulllard, Pierre, Eric Gailledrat, Pierre Moret et Feliciana Sala, Fouilles de la Rabita de Guardamar del Segura (Alicante), II, L'établissement protohistorique de La Fonteta (fin VIIIefin VIe siècle av.J.-C.), Coll. de la Casa de Velázquez, Madrid, 2006.

Ruiz Mata, Diego et Carmen Pérez, El poblado fenicio del Castillo de Doña Blanca (El Puerto de Santa María, Cádiz), El Puerto de Santa María, 1995.

Sala Sellés, Feliciana, a Algunas reflexiones sobre la fase antigua de la Contestania ibérica : de la tradición orientalizante al periodo clásico ", Anales de Arqueología Cordobesa, 7, 1996, pp. 9-31.

WaGNer, Carlos González, « Approximación al proceso histórico de Tartessos ", AEspA, 56, 1983, pp. 3-36.

WaGner, Carlos González, "Comercio lejano, colonización e intercambio desigual en la expansión fenicia arcaica por el Mediterráneo , dans Intercambio y Comercio Preclásico en el Mediterráneo, I Coloquio del CEFYD (Madrid, 1998), Madrid, 2000, pp.79-91.

WaGner, Carlos González et Jaime Alvar, a Fenicios en Occidente : la colonización agrícola , RStFen, XVII, 1, pp. 65-76.

WaGner, Carlos González et Jaime Alvar, "La colonización agrícola en la península ibérica. Estado de la cuestión y nuevas perspectivas ", dans Gómez Bellard (éd.), Ecohistoria, pp. 187-204.

WhitTAKER, C. R. "Carthaginian imperialism in the fifth and fourth centuries", dans P. D. A. Garnsey et C. R. WhitTaKer (éds.), Imperialism in the Ancient World, Cambridge, 1978, p. $59-90$. 